\title{
Characteristics of Psychiatric Hospital Work Environment Found Attractive by Professional Nurse Administrators in Japan
}

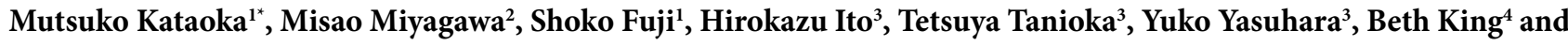 \\ Rozzano Locsin ${ }^{3}$ \\ ${ }^{1}$ Mifune Hospital, 366, Kubara-cho, Marugame-shi, Kagawa, 763-0073, Japan \\ ${ }^{2}$ Depertment of Nursing, Faculty of Health and Welfare, Tokushima Bunri University, Japan \\ ${ }^{3}$ Department of Nursing, Institute of Biomedical Sciences, Tokushima University Graduate School, Japan \\ ${ }^{4}$ Christine E. Lynn College of Nursing, Florida Atlantic University, 777 Glades Road, Boca Raton, Florida, USA
}

\begin{abstract}
Purpose: The purpose of this study is to identify characteristics of work environments in psychiatric hospitals which are appealing to nurses. Understanding characteristics of work environments of psychiatric hospital nurses can lead to attracting nurses to work in this environment, thereby increasing human resources and alleviating nurse-staffing problems.

Methods: Questionnaire copies focusing on staffing shortages of nurses in psychiatric hospitals were provided to 277 psychiatric hospital nurse administrators in Japan from May-April 2016. Data analyses included descriptive and multivariate statistics. Factor analysis together with the Kaiser-Meyer-Olkin and the Bartlett's test of Sphericity were used to assess adaptive validity. Factor loadings were set at 0.4 or more for the configuration of items, while internal consistency and reliability of the assessment data scores were done using Cronbach's alpha coefficient. Mean factor points were calculated and student's t-test and one-way analysis of variance were performed. Significance probability level was set at 0.001 . Results: Factor analysis revealed nine factors: (1) Educational system, (2) Reputation at hospitals, (3) Support system for acquisition of certificate of clinical nurse specialist/certified nurse, (4) Reputation at hospitals as places of employment, (5) Knowledge of nursing philosophy and of culture of working places, (6) Reputation of teaching for students' clinical practice, (7) Working hours and leave privileges, (8) Salary and other allowances, (9) Support system for license acquisition. Data revealed that nurse administrators who employed new graduate registered nurses found their hospitals' attractive points were: educational system, support system to acquire certificate of clinical nurse specialist, reputation of hospital as place of employment, knowledge of nursing philosophy and culture of working places, teaching students' clinical practice, and salary and other allowances.

Conclusion: Nurse administrators who have no difficulty recruiting nursing staff recognized that their hospital environments were considered appealing based on working hours and leave privileges.
\end{abstract}

\section{Introduction}

Today's global nursing shortage is having an adverse impact on health systems around the world [1]. An insufficient supply of nurses, considered essential personnel, is a critical stressor for hospitals. Many hospitals are struggling with a nurse shortage. May, Bazzoli and Gerland [2] reported that the nurse shortages resulted from a combination of factors; Nurses salary, nurses' work environment, and long-term strategies for nurse education, student nurse and nurse faculty. The relationship between nurses' working conditions, such as high workload, and job dissatisfaction can lead to low morale, absenteeism, turnover, and poor job performance. In addition, prolonged shortages may also cause reduction in patient population, potentially threaten quality patient care, and organizational effectiveness $[3,4]$.

Japan is purported to have the highest proportion of elderly citizens in comparison to other nations [5]. Japan is the most aged society in the world, with $25.0 \%$ of the population more than 65 years old in 2013 , and this rate is projected to increase to $40 \%$ in 2060 , and the increase of "oldest-old", aged 75 and more would more than double from $11 \%$ in 2010 to $27 \%$ in 2060 [6,7,8]. As a result, Japan has been suffering from a chronic shortage of nurses in terms of both quality and quantity [9].

In addition, a rapid increase in demand for nurses caused by the criterion of medical payment system (the nursing system of one nurse for every seven inpatients) in $2006 \overline{\text { has impacted the nursing shortage }}$ [10]. Along with the advanced health care and increasingly severely-ill with aging such as heart disease, cancer, and diabetes, the demand for nursing personnel is exceeding the supply and nurse staffing shortages are expected to continue.

A critical shortage of nurses is most evident in psychiatric hospitals. According to a recent survey of 937 private psychiatric hospitals in Japan [11], the employment-rate of nursing staff was $82 \%$. Of those employed, $8.9 \%$ were new graduates and $91.1 \%$ were experienced registered nurses/practical nurses who have work experience in other medical facilities/hospitals. These statistics indicate that only a small percentage of new graduates are choosing to work in psychiatric hospitals.

"Corresponding Author: Mutsuko Kataoka, Mifune Hospital, 366, Kubara-cho Marugame-shi, Kagawa, 763-0073, Japan, Tel: +81-877-23-2341, Fax: +81-87723-2344; E-mail:m1 kataoka@hotmail.com

Citation: Kataoka M, Miyagawa M, Fuji S, Ito H, Tanioka T, et al. (2016) Characteristics of Psychiatric Hospital Work Environment Found Attractive by Professional Nurse Administrators in Japan. Int J Nurs Clin Pract 3: 207. doi https://doi.org/10.15344/2394-4978/2016/207

Copyright: (C) 2016 Kataoka et al. This is an open-access article distributed under the terms of the Creative Commons Attribution License, which permits unrestricted use, distribution, and reproduction in any medium, provided the original author and source are credited. 
Citation: Kataoka M, Miyagawa M, Fuji S, Ito H, Tanioka T, et al. (2016) Characteristics of Psychiatric Hospital Work Environment Found Attractive by Professional Nurse Administrators in Japan. Int J Nurs Clin Pract 3: 207. doi: https://doi.org/10.15344/2394-4978/2016/207

Page 2 of 9

Recruitment of new graduate nurses who are passionate about caring for patients with psychiatric conditions has reached a critical point. Employing experienced psychiatric nurses, let alone those nurses who have recently graduated to work in the practice of psychiatric nursing has been difficult [12]. Efforts must be made to ensure a work environment that values and nurtures nurses and their highly skilled nursing ability. The recruitment of new nurses to practice in psychiatric nursing is more vital today than ever before.

Psychiatric nursing is an area in which achievement of a high level of expertise requires specialized knowledge and skills to address the problems of mentally-ill patients $[13,14]$. It requires years to get the specialized skills, an incentive that young nurses can be offered, including opportunities to get specialized skills of psychiatric nursing is necessary $[15,16,17]$. In addition, creative work environments to advance careers of young generation nurses could be another incentive.

Recently-graduated psychiatric mental health nurses are unsure about the skills and knowledge expected of them to practice at levels of preparations acquired in their undergraduate courses [18]. Nurses working in psychiatric hospitals need to acquire skills of therapeutic communication and empathy, and have higher levels of caring [19] The closure of many psychiatric institutions and their substitution with community-based settings, signified that health professionals should perform a variety of new duties, exhibit new skills and develop new perceptions about their work. For such alterations to occur, education plays a vital role in the re-conceptualization of psychiatric nursing and practical preparation that reinforce the shift from institutionalized work to deinstitutionalization [20]. Psychiatric mental health nurses are at the forefront of care and treatment within acute inpatient settings.

In addition, forensic psychiatric nursing requires specific personal attributes and new competencies that extend beyond traditional psychiatric nursing skills. Forensic nurses must be confident in their clinical skills, demonstrate strong leadership capabilities, and be tolerant of the uncertainties associated with these unique environments [21]. Current societal and healthcare system trends highlight the need to transform nursing education to prepare nurses capable of outstanding practice. Nurse administrators and clinical educators have to examine current practice and possibly modify aspects to include more experiential learning [22]. Increased clinical hours are required to get those specialized expert nursing skills. Understanding these situations, expert nurses act naturally without explicitly making decisions and solving problems [23, 24]. Because of these needed additional skills, in order to attract recently-graduated and young psychiatric nurses, it is necessary that educational opportunities are offered to them.

Therefore, it is important to know the characteristics of the work environments of psychiatric hospitals which do not have problems of nurse staffing shortages versus those psychiatric hospitals which have the problem of nurse staffing shortages. Findings from this research study can provide information to facilitate work environment changes to attract new nursing graduates to for employment in psychiatric hospitals.

\section{Purpose of the Study}

The aim of this study was to identify characteristics of the work environment in psychiatric hospitals that are attractive to nurses.

\section{Methods}

\section{Data collection}

In this cross-sectional study, purposive sampling was used to select 277 hospitals from the 1208 member hospitals of the Japan Psychiatric Hospitals Association. Psychiatric hospitals in the following six categories were included in the study: 14 hospitals with less than 100 beds, 98 hospitals with between 100 and 199 beds, 82 hospitals with between 200 and 299 beds, 43 hospitals with between 300 and 399 beds, 23 hospitals with between 400 and 499 beds, and 17 hospitals with more than 500 beds.

The sample consisted of 277 nurse administrators at psychiatric hospitals who consented to participate in the study, following an initial telephone call to enhance cooperation to participate in the study. Questionnaires were returned by 234 participants, with a return rate of 84\%. Data was collected from April 2016 to May 2016.

\section{Survey questionnaire instrument}

The questionnaire consisted of two parts: (1) demographics related to the psychiatric hospital and employed nursing staff and (2) questions related to nursing staff shortage. Questions related to the psychiatric hospital demographics included:

(1) Basic hospital information: size of population of cities, counties where hospitals are located; number of operating beds; bed occupancy rate; number of outpatients to the psychiatric department per day (Including patients of psychiatric day-care; acceptance of students' clinical practice from the nursing university, practical nurse school, and registered nurse school; average age, oldest age of nursing staffs; difficulty of recruiting; and the number of new graduates, number of those employed by their home hospital by a support of nursing license acquisition, number of experienced employed persons in the year of 2015.

(2) The second component of the questionnaire was developed from data derived from previous studies [25, 26]. The questionnaire consisted of 53 questions related to the nursing staffing shortage in psychiatric hospitals. The responses were based on a 5-point Likert scale (strongly agree, agree, neutral, disagree, and strongly disagree).

\section{Definition of terms used in the questionnaire}

In this study, the questionnaire consisted of item statements regarding the nursing staff. These nursing staff were identified as (a). Regular nursing staff members are full time registered nurses/ practical nurses, however, part-time staff and temporary workers were excluded in this study. (b). New graduates were defined as registered nurses/practical nurses employed in the same year they were licensed as registered nurse/practical nurse. They were employed without any nursing experience. (c). In general, employed persons were registered nurses/practical nurses who were supported by their home hospitals to obtain a nursing license. They worked for the hospital as a practical nurse/nurse's aide while they studied to obtain registered nurse/ practical nurse licenses, and continued working at the same hospital. (d). Experienced employed persons were registered nurses/practical nurses who have work experience in other medical facilities/hospitals.

\section{Data analysis}

Data analysis used descriptive and multivariate statistics to determine the distribution of survey items pertaining to staffing 
Citation: Kataoka M, Miyagawa M, Fuji S, Ito H, Tanioka T, et al. (2016) Characteristics of Psychiatric Hospital Work Environment Found Attractive by Professional Nurse Administrators in Japan. Int J Nurs Clin Pract 3: 207. doi: https://doi.org/10.15344/2394-4978/2016/207

Page 3 of 9

shortage of nursing in psychiatric hospitals in Japan. Factor analysis (principal factor analysis, Varimax rotation without Kaiser Normalization) was used in order to determine the factors together with the Kaiser-Meyer-Olkin (KMO) and the Bartlett's test of Sphericity to assess the adaptive validity of the factor analysis. Factor loadings were set at 0.4 or more for the configuration of items. Mean factor points were calculated excluding missing values. Factor loadings of less than 0.4 were omitted. Internal consistency and reliability of the assessment data scores was done using Cronbach's alpha coefficient.

Responses were re-categorized as follows: (1) the number of recently employed nursing staff who responsed with zero were categorized into group "Did not" and more than one into group "Employed"; (2) the status of recruitment for nursing staffs, responses of "not distressed" and "rarely distressed" were categorized into group "No difficulty", (3) "while responses of much distressed" and "a little distressed" were categorized into group "Difficulty."

Welch's t-test with test for equality of variance was used to compare descriptions between two groups. Welch's one-way analysis of variance (ANOVA) with test for equality of variance were used among the category of number of operating beds, and category of number of outpatient visits. Post hoc analysis was performed with of GamesHowell test.

Significance probability level was set at 0.001 . All statistical analyses were performed using the SPSS for Windows software (version 11.0; SPSS Inc., Chicago).

\section{Ethical considerations}

The University of Tokushima Hospital Clinical Study Ethical Review Board approved this study (No. 2430). Return of the survey questionnaire implied that the participants gave consent to participate in the study. The cover letter provided information regarding privacy protection, confidentiality, anonymity, and that only aggregate data would be used in reporting the findings.

\section{Results}

\section{Characteristics of the hospitals}

Table 1(a) shows characteristics of the psychiatric hospitals. There were 143 hospitals $(61.1 \%)$ that accepted students from the Registered Nurse School, or Nursing University to have their clinical practice. However, 54 of these hospitals (23.1\%) had students' clinical practice from practical nurse schools.

Table 1(b) shows average age of the registered nurse/practical nurse according to the number of hospital beds. Maximum age of registered nurses was 86 years, and practical nurses was 79 years.

\section{Results of the factor analysis (Table 2)}

A factor analysis was conducted on the results of the 53 item questionnaire. Data analysis yielded 9 factors ( 35 items). Their alpha

\begin{tabular}{|c|c|c|c|}
\hline Items & Classification & $\mathrm{n}$ & $\%$ \\
\hline \multirow{3}{*}{$\begin{array}{l}\text { Regarding the size of population in cities (hospitals are } \\
\text { located) }\end{array}$} & Less than 50,000 & 43 & 18.4 \\
\hline & Between 50,000 and 100,000 & 41 & 17.5 \\
\hline & No less than 100,000 & 147 & 62.8 \\
\hline \multirow[t]{4}{*}{ The number of operating beds } & Less than 100 beds & 15 & 6.4 \\
\hline & Between 100 and 199 beds & 89 & 38.0 \\
\hline & Between 200 and 299 beds & 66 & 28.2 \\
\hline & No less than 300 beds & 64 & 27.4 \\
\hline \multirow[t]{4}{*}{ The bed occupancy rate } & Less than $85 \%$ & 36 & 15.4 \\
\hline & Between 85 and $90 \%$ & 58 & 24.8 \\
\hline & Between 90 and $95 \%$ & 84 & 35.9 \\
\hline & No less than $95 \%$ & 53 & 22.6 \\
\hline \multirow[t]{4}{*}{ The number of outpatient visits } & Less than 50 out patient visits & 69 & 29.5 \\
\hline & Between 50 and 100 outpatient visits & 96 & 41.0 \\
\hline & Between 100 and 150 outpatient visits & 34 & 14.5 \\
\hline & No less than 150 outpatiens visits & 35 & 15.0 \\
\hline
\end{tabular}

\begin{tabular}{|c|c|c|c|c|c|c|c|c|c|}
\hline \multirow[t]{2}{*}{ Type of license } & \multirow{2}{*}{$\begin{array}{l}\text { Average age } \\
\text { The number of hospital beds }\end{array}$} & \multicolumn{2}{|c|}{30 's } & \multicolumn{2}{|c|}{40 's } & \multicolumn{2}{|c|}{50 's } & \multicolumn{2}{|c|}{ Over 60's } \\
\hline & & $\mathrm{n}$ & $\%$ & $\mathrm{n}$ & $\%$ & $\mathrm{n}$ & $\%$ & $\mathrm{n}$ & $\%$ \\
\hline \multirow[t]{5}{*}{ Registered nurses } & Less than 100 beds & 1 & 6.7 & 10 & 66.7 & 4 & 26.7 & 0 & 0 \\
\hline & Between 100 and 199 beds & 5 & 5.7 & 63 & 72.4 & 18 & 20.7 & 1 & 1.1 \\
\hline & Between 200 and 299 beds & 5 & 8.1 & 47 & 75.8 & 9 & 14.5 & 1 & 1.6 \\
\hline & No less than 300 beds & 12 & 19.7 & 44 & 72.1 & 4 & 6.6 & 1 & 1.6 \\
\hline & Total & 23 & 10.2 & 164 & 72.9 & 35 & 15.6 & 3 & 1.3 \\
\hline \multirow[t]{5}{*}{ Practical nurses } & Less than 100 beds & 1 & 6.7 & 10 & 66.7 & 4 & 26.7 & 0 & 0 \\
\hline & Between 100 and 199 beds & 4 & 4.7 & 49 & 57.0 & 32 & 37.2 & 1 & 1.2 \\
\hline & Between 200 and 299 beds & 4 & 6.5 & 38 & 61.3 & 19 & 30.6 & 1 & 1.6 \\
\hline & No less than 300 beds & 6 & 10.0 & 35 & 58.3 & 18 & 30.0 & 1 & 1.7 \\
\hline & Total & 15 & 6.7 & 132 & 59.2 & 73 & 32.7 & 3 & 1.3 \\
\hline
\end{tabular}

Table 1(b): Average age distribution of the registered nurse/practical nurse. 
Citation: Kataoka M, Miyagawa M, Fuji S, Ito H, Tanioka T, et al. (2016) Characteristics of Psychiatric Hospital Work Environment Found Attractive by Professional Nurse Administrators in Japan. Int J Nurs Clin Pract 3: 207. doi: https://doi.org/10.15344/2394-4978/2016/207

Page 4 of 9

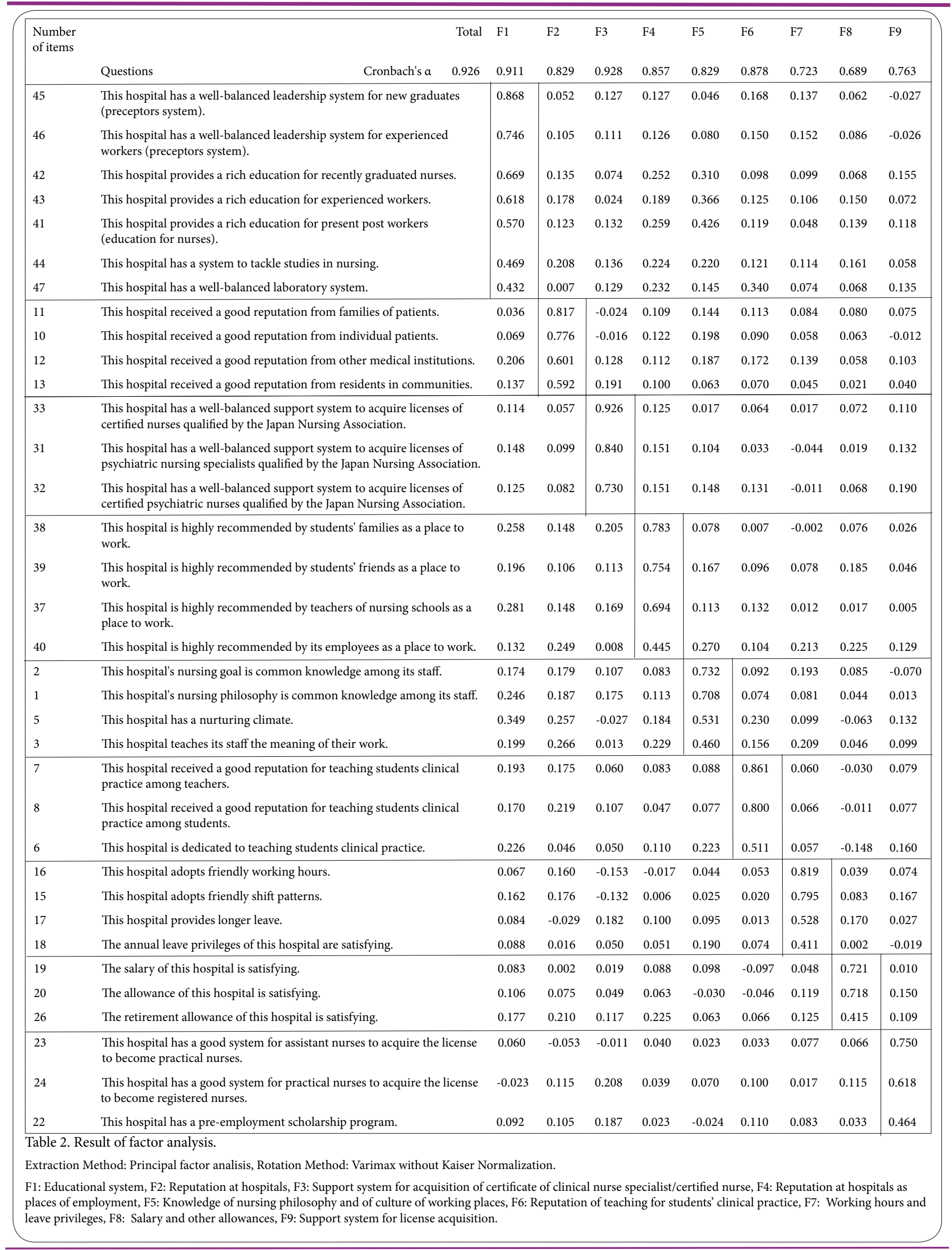


Citation: Kataoka M, Miyagawa M, Fuji S, Ito H, Tanioka T, et al. (2016) Characteristics of Psychiatric Hospital Work Environment Found Attractive by Professional Nurse Administrators in Japan. Int J Nurs Clin Pract 3: 207. doi: https://doi.org/10.15344/2394-4978/2016/207

Page 5 of 9

\begin{tabular}{|c|c|c|c|c|c|c|c|c|c|c|c|c|c|c|c|}
\hline \multirow[t]{4}{*}{ Factor } & & \multicolumn{6}{|c|}{ Registered nurses } & \multicolumn{6}{|c|}{ Practical nurses } & \multicolumn{2}{|c|}{$\begin{array}{l}\text { The difficulty in employment } \\
\text { of nursing staff }\end{array}$} \\
\hline & & \multicolumn{2}{|c|}{ A } & \multicolumn{2}{|c|}{ B } & \multicolumn{2}{|c|}{ C } & \multicolumn{2}{|c|}{ A } & \multicolumn{2}{|c|}{ B } & \multicolumn{2}{|c|}{ C } & \multirow[b]{2}{*}{ No difficulty } & \multirow[b]{2}{*}{ Difficulty } \\
\hline & & Employed & Did not & Employe & Did not & Employe & Did not & Employec & Did not & Employe & Did not & Employe & Did not & & \\
\hline & $\mathrm{n}$ & 94 & 133 & 86 & 140 & 194 & 28 & 49 & 178 & 69 & 157 & 147 & 78 & 62 & 168 \\
\hline \multirow{4}{*}{ F1 } & Mean & 3.623 & 2.607 & 3.370 & 2.807 & 3.025 & 2.943 & 3.236 & 2.970 & 3.187 & 2.949 & 2.913 & 3.212 & 3.325 & 2.920 \\
\hline & $\pm \mathrm{SD}$ & 0.884 & 0.963 & 0.875 & 1.099 & 1.087 & 0.862 & 0.997 & 1.067 & 0.969 & 1.084 & 1.009 & 1.114 & 1.106 & 1.022 \\
\hline & $\mathrm{t}$-value & \multicolumn{2}{|c|}{-8.098} & \multicolumn{2}{|c|}{-4.255} & \multicolumn{2}{|c|}{-0.383} & \multicolumn{2}{|c|}{-1.564} & \multicolumn{2}{|c|}{-1.568} & \multicolumn{2}{|c|}{2.045} & \multicolumn{2}{|c|}{-2.605} \\
\hline & $\mathrm{p}$-value & \multicolumn{2}{|c|}{$* * *$} & & & & & & & & & & & & \\
\hline & Mean & 3.543 & 3.355 & 3.462 & 3.413 & 3.430 & 3.429 & 3.439 & 3.431 & 3.391 & 3.449 & 3.379 & 3.522 & 3.528 & 3.388 \\
\hline & $\pm \mathrm{SD}$ & 0.472 & 0.541 & 0.475 & 0.549 & 0.538 & 0.390 & 0.488 & 0.531 & 0.543 & 0.512 & 0.538 & 0.477 & 0.536 & 0.506 \\
\hline F2 & $\mathrm{t}$-value & -2 & & & & & & & & & & & & & \\
\hline & $\mathrm{p}$-value & $\mathrm{n}$ & & & & & & & & & & & & & n.s. \\
\hline & Mean & 2.582 & 1.832 & 2.407 & 1.988 & 2.169 & 2.000 & 1.953 & 2.195 & 2.174 & 2.136 & 2.075 & 2.287 & 2.183 & 2.099 \\
\hline & $\pm \mathrm{SD}$ & 1.413 & 1.197 & 1.347 & 1.317 & 1.357 & 1.311 & 1.466 & 1.303 & 1.334 & 1.348 & 1.373 & 1.284 & 1.368 & 1.337 \\
\hline F3 & $\mathrm{t}$-value & -4 & & & & & & & & & & & & & \\
\hline & $\mathrm{p}$-value & *** & & & & & & & & & & & & & \\
\hline & Mean & 3.306 & 2.412 & 3.093 & 2.584 & 2.778 & 2.786 & 2.893 & 2.751 & 3.025 & 2.669 & 2.670 & 2.987 & 3.109 & 2.628 \\
\hline $\mathrm{F} 4$ & $\pm \mathrm{SD}$ & 0.792 & 1.012 & 0.905 & 1.049 & 1.060 & 0.841 & 0.920 & 1.052 & 0.759 & 1.107 & 1.050 & 0.954 & 1.015 & 1.016 \\
\hline & $\mathrm{t}$-value & -7. & & & & & & & & & & & & -3.187 & \\
\hline & $\mathrm{p}$-value & ** & & & & & & & & & & & & n.s. & \\
\hline F5 & Mean & 3.758 & 3.406 & 3.625 & 3.504 & 3.549 & 3.589 & 3.694 & 3.513 & 3.540 & 3.554 & 3.483 & 3.676 & 3.831 & 3.464 \\
\hline & $\pm \mathrm{SD}$ & 0.687 & 0.758 & 0.691 & 0.782 & 0.780 & 0.519 & 0.676 & 0.764 & 0.732 & 0.759 & 0.737 & 0.767 & 0.777 & 0.718 \\
\hline & $\mathrm{t}$-value & -3 & & & & & & -1.505 & & 0.132 & & & & & \\
\hline & $\mathrm{p}$-value & ** & & & & & & n.s. & & n.s. & & & & & \\
\hline & Mean & 3.649 & 2.098 & 3.302 & 2.381 & 2.737 & 2.654 & 3.429 & 2.550 & 3.130 & 2.556 & 2.653 & 2.863 & 3.102 & 2.595 \\
\hline F6 & $\pm \mathrm{SD}$ & 1.477 & 1.938 & 1.679 & 1.976 & 1.953 & 1.705 & 1.561 & 1.967 & 1.793 & 1.950 & 1.917 & 1.931 & 1.891 & 1.907 \\
\hline & $\mathrm{t}$-value & -6. & & & & & & & & & & & & & \\
\hline & $\mathrm{p}$-value & *** & & & & & & & & & & & & & \\
\hline F7 & Mean & 4.080 & 3.947 & 4.035 & 3.977 & 4.021 & 3.893 & 4.041 & 3.992 & 3.957 & 4.018 & 3.988 & 4.029 & 4.310 & 3.905 \\
\hline & $\pm \mathrm{SD}$ & 0.616 & 0.697 & 0.622 & 0.693 & 0.663 & 0.712 & 0.650 & 0.672 & 0.603 & 0.693 & 0.667 & 0.666 & 0.638 & 0.645 \\
\hline & $\mathrm{t}$-value & -1.478 & & 636 & & 945 & & & & & & & & & \\
\hline & $\mathrm{p}$-value & n.s. & & s. & & 1.s. & & & & & & & & & \\
\hline F8 & Mean & 2.993 & 2.697 & 2.845 & 2.800 & 2.832 & 2.726 & 2.802 & 2.824 & 2.798 & 2.826 & 2.746 & 2.948 & 3.021 & 2.748 \\
\hline & $\pm \mathrm{SD}$ & 0.601 & 0.650 & 0.666 & 0.636 & 0.654 & 0.636 & 0.608 & 0.657 & 0.595 & 0.669 & 0.644 & 0.638 & 0.708 & 0.602 \\
\hline & $\mathrm{t}$-value & -3. & & & & & & & & & & & & & \\
\hline & $\mathrm{p}$-value & ** & & & & & & & & & & & & & \\
\hline F9 & Mean & 3.422 & 2.920 & 3.662 & 2.805 & 3.110 & 3.191 & 3.252 & 3.093 & 3.647 & 2.904 & 3.038 & 3.295 & 3.011 & 3.173 \\
\hline & $\pm \mathrm{SD}$ & 1.234 & 1.594 & 1.152 & 1.560 & 1.526 & 1.195 & 1.487 & 1.473 & 1.080 & 1.570 & 1.549 & 1.329 & 1.310 & 1.520 \\
\hline & $\mathrm{t}$-value & -2. & & & & & & & & & & & & & \\
\hline & $\mathrm{p}$-value & $\mathrm{n}$ & & & & & & & & & & & & & \\
\hline
\end{tabular}

Table 3. Comparison of hospitals regarding whether or not they employed employees, and hospitals had difficulties in the recruitment.

Analysis method: Welch's t-test, $\mathrm{SD}=$ Standard Deviation, ${ }^{* * *}: \mathrm{p}<0.001$, n.s.: not significant.

F1: Educational system, F2: Reputation at hospitals, F3: Support system for acquisition of certificate of clinical nurse specialist/certified nurse, F4: Reputation at hospitals as places of employment, F5: Knowledge of nursing philosophy and of culture of working places, F6: Reputation of teaching for students' clinical practice, F7: Working hours and leave privileges, F8: Salary and other allowances, F9: Support system for license acquisition.

A: The new graduates are registered nurses/practical nurses employed in the same year they were licensed as registered nurse/practical nurse. They were employed without any nursing experience.

B:Employed persons are registered nurses/practical nurses who were supported by their home hospitals to obtain a nursing license. They are those who worked for the hospital as a practical nurse/nurse's aide while they studied to obtain registered nurse/practical nurse licenses, and continued working at the same hospital.

C:Experienced employed persons are registered nurses/practical nurses who have work experience in other medical facilities/hospitals. 
Citation: Kataoka M, Miyagawa M, Fuji S, Ito H, Tanioka T, et al. (2016) Characteristics of Psychiatric Hospital Work Environment Found Attractive by Professional Nurse Administrators in Japan. Int J Nurs Clin Pract 3: 207. doi: https://doi.org/10.15344/2394-4978/2016/207

Page 6 of 9

\begin{tabular}{|c|c|c|c|c|c|c|c|c|c|}
\hline \multicolumn{2}{|c|}{ Factors } & \multicolumn{4}{|c|}{ The number of operating beds } & \multicolumn{4}{|c|}{ The number of outpatient visits } \\
\hline & & $\mathrm{a}$ & $\mathrm{b}$ & c & $\mathrm{d}$ & a & $\mathrm{b}$ & c & $\mathrm{d}$ \\
\hline & & $\begin{array}{c}\text { Less than } 100 \\
\text { beds }\end{array}$ & $\begin{array}{l}\text { Between } 100 \\
\text { and } 199 \text { beds }\end{array}$ & $\begin{array}{c}\text { Between } \\
200 \text { and } 299 \\
\text { beds }\end{array}$ & $\begin{array}{c}\text { No less than } 300 \\
\text { beds }\end{array}$ & $\begin{array}{l}\text { Less than } 50 \text { out } \\
\text { patient visits }\end{array}$ & $\begin{array}{c}\text { Between } 50 \text { and } \\
100 \text { outpatient } \\
\text { visits }\end{array}$ & $\begin{array}{l}\text { Between } 100 \text { and } \\
150 \text { outpatient } \\
\text { visits }\end{array}$ & $\begin{array}{l}\text { No less than } 150 \\
\text { outpatiens visits }\end{array}$ \\
\hline & $\mathrm{n}$ & 15 & 89 & 66 & 64 & 69 & 96 & 34 & 35 \\
\hline \multirow{5}{*}{ F1 } & Mean & 2.410 & 2.610 & 3.186 & 3.612 & 2.516 & 3.137 & 3.521 & 3.298 \\
\hline & $\pm \mathrm{SD}$ & 0.700 & 1.045 & 1.001 & 0.875 & 1.151 & 0.914 & 0.773 & 1.109 \\
\hline & F-value & \multicolumn{4}{|c|}{15.850} & \multicolumn{4}{|c|}{9.977} \\
\hline & $\mathrm{p}$-value & \multicolumn{4}{|c|}{$* * *$} & \multicolumn{4}{|c|}{$* * *$} \\
\hline & & \multicolumn{4}{|c|}{$\mathrm{a}<\mathrm{c}, \mathrm{d} \quad \mathrm{b}<\mathrm{c}, \mathrm{d}$} & \multicolumn{4}{|c|}{$\mathrm{a}<\mathrm{b}, \mathrm{c}, \mathrm{d}$} \\
\hline \multirow{4}{*}{$\mathrm{F} 2$} & Mean & 3.367 & 3.402 & 3.402 & 3.504 & 3.326 & 3.430 & 3.544 & 3.507 \\
\hline & $\pm \mathrm{SD}$ & 0.462 & 0.561 & 0.476 & 0.507 & 0.504 & 0.505 & 0.517 & 0.554 \\
\hline & F-value & \multicolumn{4}{|c|}{0.663} & \multicolumn{4}{|c|}{1.760} \\
\hline & F-value & \multicolumn{4}{|c|}{ n.s. } & \multicolumn{4}{|c|}{ n.s. } \\
\hline \multirow{5}{*}{ F3 } & Mean & 1.867 & 1.727 & 2.268 & 2.609 & 1.643 & 2.170 & 2.412 & 2.705 \\
\hline & $\pm \mathrm{SD}$ & 1.356 & 1.203 & 1.308 & 1.373 & 1.298 & 1.279 & 1.054 & 1.508 \\
\hline & F-value & \multicolumn{4}{|c|}{6.300} & \multicolumn{4}{|c|}{6.155} \\
\hline & $\mathrm{p}$-value & \multicolumn{4}{|c|}{$* * *$} & \multicolumn{4}{|c|}{$* * *$} \\
\hline & & \multicolumn{4}{|c|}{$\mathrm{b}<\mathrm{c}, \mathrm{d}$} & \multicolumn{4}{|c|}{$\mathrm{a}<\mathrm{c}, \mathrm{d}$} \\
\hline & Mean & 2.000 & 2.438 & 2.985 & 3.152 & 2.341 & 2.844 & 3.103 & 3.021 \\
\hline F4 & $\pm \mathrm{SD}$ & 1.150 & 1.037 & 0.898 & 0.905 & 1.169 & 0.912 & 0.791 & 1.033 \\
\hline 17 & F-value & & & 72 & & & & & \\
\hline & $\mathrm{p}$-value & & & & & & & & \\
\hline & & & $\mathrm{a}<\mathrm{c}, \mathrm{d}$ & $\mathrm{b}<\mathrm{c}, \mathrm{d}$ & & & & $\mathrm{c}, \mathrm{d}$ & \\
\hline & Mean & 3.433 & 3.396 & 3.633 & 3.758 & 3.413 & 3.552 & 3.868 & 3.600 \\
\hline F5 & $\pm \mathrm{SD}$ & 0.530 & 0.735 & 0.742 & 0.769 & 0.772 & 0.736 & 0.619 & 0.777 \\
\hline & F-value & & & & & & & & \\
\hline & $\mathrm{p}$-value & & & & & & & & \\
\hline & Mean & 1.178 & 1.955 & 3.207 & 3.734 & 1.812 & 2.993 & 3.235 & 3.429 \\
\hline & $\pm \mathrm{SD}$ & 1.603 & 1.980 & 1.703 & 1.368 & 1.999 & 1.713 & 1.748 & 1.837 \\
\hline F6 & F-value & & & & & & & & \\
\hline & $\mathrm{p}$-value & & & & & & & & \\
\hline & & & $\mathrm{a}<\mathrm{c}, \mathrm{d}$ & $\mathrm{b}<\mathrm{c}, \mathrm{d}$ & & & & $c, d$ & \\
\hline & Mean & 3.850 & 3.899 & 4.061 & 4.148 & 4.036 & 3.987 & 4.147 & 3.886 \\
\hline F7 & $\pm \mathrm{SD}$ & 0.625 & 0.686 & 0.619 & 0.668 & 0.720 & 0.593 & 0.616 & 0.773 \\
\hline & F-value & & & & & & & & \\
\hline & $\mathrm{p}$-value & & & & & & & & \\
\hline & Mean & 2.578 & 2.689 & 2.864 & 3.005 & 2.763 & 2.788 & 2.833 & 2.990 \\
\hline F8 & $\pm \mathrm{SD}$ & 0.684 & 0.662 & 0.608 & 0.583 & 0.679 & 0.618 & 0.721 & 0.521 \\
\hline & F-value & & & & & & & & \\
\hline & $\mathrm{p}$-value & & & & & & & & \\
\hline & Mean & 2.111 & 2.704 & 3.490 & 3.594 & 2.937 & 3.115 & 3.529 & 3.171 \\
\hline & $\pm \mathrm{SD}$ & 1.833 & 1.505 & 1.228 & 1.305 & 1.647 & 1.468 & 1.048 & 1.456 \\
\hline F9 & F-value & & & & & & & & \\
\hline & $\mathrm{p}$-value & & & & & & & & \\
\hline & & & $a<d$ & $\mathrm{~b}<\mathrm{c}, \mathrm{d}$ & & & & & \\
\hline
\end{tabular}

Table 4. Comparison of categories based on the number of operating beds, and the number of outpatients.

Analysis method: Test for equality of variance, Welch's one-way analysis of variance (ANOVA): Post hoc analysis was performed with of Games-Howell test. ${ }^{* * *}: \mathrm{p}<0.001, \mathrm{SD}=$ Standard Deviation, n.s.: not significant.

F1: Educational system, F2: Reputation at hospitals, F3: Support system for acquisition of certificate of clinical nurse specialist/certified nurse, F4: Reputation at hospitals as places of employment, F5: Knowledge of nursing philosophy and of culture of working places, F6: Reputation of teaching for students' clinical practice, F7: Working hours and leave privileges, F8: Salary and other allowances, F9: Support system for license acquisition. 
Citation: Kataoka M, Miyagawa M, Fuji S, Ito H, Tanioka T, et al. (2016) Characteristics of Psychiatric Hospital Work Environment Found Attractive by Professional Nurse Administrators in Japan. Int J Nurs Clin Pract 3: 207. doi: https://doi.org/10.15344/2394-4978/2016/207

Page 7 of 9

factors were in the range of 0.689 to 0.928 . This showed that the overall data obtained high internal consistency for each factor. The KMO sample adequacy was 0.84 and the Bartlett's test of Sphericity was $\mathrm{p}<0.001$; indicating the questionnaire was a reliable instrument.

The 9 factors are discussed below.

(Factor 1) The first factor, the educational system (hereafter shown as F1) consisted of the following seven items: leadership system for new graduates; leadership system for experienced workers; education for recently-graduated nurses; education for experienced workers; education for present post workers; system of study in nursing; and laboratory work system.

(Factor 2) The second factor, reputation at hospitals (F2) consisted of the following four items: patients' family; individual patients, other medical institutions; and by the residents in communities.

(Factor 3) The third factor, support system for acquisition of certificate of clinical nurse specialist/certified nurse (F3) consisted of the following three items: certified nurses; psychiatric nurses; and certified psychiatric nurses.

(Factor 4) The fourth factor, reputation at hospitals as places of employment (F4) consisted of the following four items: recommendation by students' family; recommendation by students friends; recommendation by teachers of nursing schools; and recommendation by employees.

(Factor 5) The fifth factor, knowledge of nursing philosophy and of culture of working places (F5) consisted of the following four items: knowledge of nursing goals; knowledge of nursing philosophy; climate of nurturing someone; and meaning of work.

(Factor 6) The sixth factor, reputation of teaching for students' clinical practice (F6) consisted of the following three items; reputation by teachers; reputation by students; and dedication to clinical practice.

(Factor 7) The seventh factor, working hours and leave privileges (F7) consisted of the following four items; working hours; shift pattern; leaves; and annual leave privileges.

(Factor 8) The eighth factor, salary and other allowances (F8) consisted of the following three items; salary; allowance; and retirement allowance.

(Factor 9) The ninth factor, support system for license acquisition (F9) consisted of the following three items; license of practical nurse; license of registered nurse; and scholarship before employment.

Eighteen items were excluded in the process of factor analysis: traffic accessibility, web page, electronic health record system, career fair, child-support system, turnover rate, male staff, personal relationship in an office, maintenance of hospital lodgments, maintenance of childcare centers (nursery), exterior view, interior view of hospitals, name recognition of hospital, visits to nursing schools, information exchanged with teachers, working overtime, publicity activity for recruitment of nursing staff at lectures in nursing schools, and publicity activity for recruitment of nursing staffs at the practical training.
Comparison of hospitals regarding whether or not they employed nursing staffs, and hospitals which had difficulties in the recruitment of nursing staffs (Table 3 ).

The findings related to:

(a). the employment of new registered nurse graduates; 94 hospitals employed them, and 133 hospitals employed none. As for the six factors, F1, F3, F4, F5, F6 and F8 hospitals that employed new graduates received significantly higher scores than those that employed none $(\mathrm{p}<0.001)$.

(b). hospitals who supported registered nurses to obtain licenses: 86 hospitals responded "yes" and 140 responded "no." As for the four factors, i.e. F1, F4, F6, F9, the former showed higher scores compared to the latter $(\mathrm{p}<0.001)$.

(c), the adoption of practical nurses who got licenses by the support by their own facilities: 69 hospitals responded "yes" and 157 responded "no." F9 showed significantly higher scores in hospitals who responded "yes," compared to those who responded "no" ( $<<0.001)$.

(d). the adoption of experienced nurses: there was no significant difference between new graduates and experienced assistant nurses.

(e). the difficulty in recruitment of nursing staffs: 62 hospitals did not have difficulty and 168 hospitals have difficulty. F7 showed significantly higher scores in hospitals without difficulty in recruitment of those staff, compared to those having them $(\mathrm{p}<0.001)$. However, other factors didn't show significantly difference.

Comparison of categories based on the number of operating beds, and the number of outpatients (Table 4).

In terms of the categories based on the number of operating beds, five factors, F1, F3, F4, F6, and F9 showed a significant difference. In the post hoc test, three factors, F1, F4, and F6 showed significantly higher score more than 200 beds compared to those with less than 100 beds; and hospitals with more than 200 beds compared with less than $100 \sim 199$ beds $(\mathrm{p}<0.001)$.

F3 showed significantly higher scores that hospitals with more than 200 beds compared with less than $100 \sim 199$ beds $(\mathrm{p}<0.001)$. F9 showed significantly higher scores that hospitals with more than

300 beds compared to those with less than 100 beds; and hospitals with more than 200 beds compared with less than 100 199 beds $(\mathrm{p}<0.001$.

In the category of the number of outpatient visits, four factors, $\mathrm{F} 1, \mathrm{~F} 3, \mathrm{~F} 4$, and F6 showed a significant differences $(\mathrm{p}<0.001)$. In the post hoc test, F1, F4, and F6 showed significantly higher scores that categories of hospitals with more than 50 outpatient visits than those with less than $50(\mathrm{p}<0.001)$.

As for $\mathrm{F} 3$ categories of, hospitals with more than 100 outpatient visits showed significantly higher than those with less than $50(\mathrm{p}<0.001)$.

\section{Discussion}

The aim of this study was to identify characteristics of the work environment in psychiatric hospitals that is appealing to nurses. This study nine factors identified, seven were found to attract nurses to practice in psychiatric hospitals.

The first characteristic related to the recruitment of new graduates; Six factors (educational system, support system for acquisition of 
Citation: Kataoka M, Miyagawa M, Fuji S, Ito H, Tanioka T, et al. (2016) Characteristics of Psychiatric Hospital Work Environment Found Attractive by Professional Nurse Administrators in Japan. Int J Nurs Clin Pract 3: 207. doi: https://doi.org/10.15344/2394-4978/2016/207

Page 8 of 9

certificate of clinical nurse specialist/certified nurse, reputation at hospitals as places of employment, knowledge of nursing philosophy and of culture of working places, reputation of teaching for students clinical practice, salary and other allowances) yielded significantly higher scores from hospitals that employed new graduates than those that employed none.

Hospitals which employed registered nurses who received support from their hospital to achieve a license compared to those who were not supported, showed significantly higher scores for the following four factors: educational system, reputation at hospitals as places of employment, reputation of teaching for students' clinical practice, and support system for license acquisition. In addition, hospitals that employed practical nurses who were able to obtain a licenses with the support of their own facilities, compared to those without, showed significantly higher scores for the support system for license acquisition.

Characteristics of salary and other allowances showed significantly higher scores in hospitals without difficulty in recruitment of new graduate registered nurse, compared to those employed new registered nurses graduates. Salary and other allowances are important criteria for nurses seeking employment in a hospital. Today, websites are a very important information source for nurses searching for a new job placement. Inclusion of salaries and other allowances on hospital websites could be beneficial to the recruitment of nurses.

In this study the average age of the nursing staff, both the registered nurse and practical nurse, was 40 years old. Nearly $90 \%$ of the respondents were between the age-range of 90 to 50 years old. Maximum age of the nursing staff who worked in the surveyed hospitals, was 86 for the registered nurses, and 79 years for the practical nurse. This indicates an aging nursing workforce within psychiatric hospitals and verifies the need for attracting new registered nurse graduates to the practice of psychiatric nursing.

In addition, only $40 \%$ of the participants indicated that they employed new graduate nurses and 20\% employed new graduate practical nurses. Other employees were registered nurses and practical nurses with work experience in other medical facilities/hospitals The majority of the hospitals, $70 \%$, indicated having difficulty with recruitment. As a result, they had to secure nursing staff by the extension of retirement or by the post retirement employment, which led to the increased number of aging characteristic of nursing staff.

The factor, educational system, revealed that participation by nursing students in clinical experiences at the hospital was significant. The presence of nursing students indicate that the hospital values learning and is supportive of providing beginning registered nurses' support. Other examples of educational support is the presence of clinical nurse specialists/certified nurses in the hospital and the value a director of nursing attaches to enhancement of professional careers.

Another factor, which was significant, related to the hospital support of further license acquisition. Nursing staff who desire to enhance their careers from assistant nurses, or practical nurses, to registered nurses while continuing to work, appreciated the support of their employers. A support system for license acquisition by hospitals is considered an important factor regarding the recruitment of nursing staff. In addition, hospitals which indicated no difficulty with recruitment of nursing staff, had significantly higher scores related to the required number of working hours and leave privileges than hospitals who had difficulty with recruitment. Another factor which enhances recruitment of nurses.

Factors which have been related to nurses who quit their job are: bearing and raising children, working overtime, and difficulty in taking leave [27]. Another study indicated that important factors for employment are money, improved working conditions, refresher courses, and health insurance. Nurses value flexible working hours, part-time opportunities, consideration of family lives [28]. In response, the Japanese government has implemented the following measures and expectations for the nursing work environment: a worklife balance campaign, accelerated adoption of the short-hour regular staff system, revision of the Guidelines for Review of Working Hours concerning measures to improve the utilization of paid holidays, and amendment of the Child Care and Family Care Leave The law requires workplaces to allow workers rearing a child under three to work short hours or to excuse them from overtime work at their request [9]. It is important to develop the hospital working conditions tailored to such nursing staff needs.

Regarding the categories based on the number of operating beds, four factors (educational system, support system for acquisition of certificate of clinical nurse specialist/certified nurse, reputation at hospitals as places of employment, and reputation of teaching for students' clinical practice) had significantly higher scores for hospitals with more than 200 beds compared to hospitals with100-199 beds. Furthermore, three factors (educational system, reputation at hospitals as places of employment, reputation of teaching for students' clinical practice) showed significantly higher scores with hospitals that had more than 100 outpatient visits than those with less than 50. In addition, the factor of a support system for license acquisition was significant for hospitals where the number of beds was more than 300, compared to those with less than 100 beds, and those with more than 200 beds, compared to those with between 100 and 199 beds, showed significantly higher scores. It is supposed that nurse administrators at hospitals with a large number of beds or outpatients highly evaluated this factor. It is clear that the size of the hospitals as indicated by number of beds, is a characteristic related to the appealing recruitment factor.

\section{Recommendations}

In order to solve the registered nurse shortage, the culture at work needs improvement and, the nursing staff must experience occupational satisfaction [28]. One way to impact the work culture is through the hospitals nursing philosophy and to ensure that the nursing goals are widely known at the hospital. When nurses are aware of the vision, philosophy and goals of nursing, they can be easily integrated and the work culture can be impacted. If the work culture is excellent, the hospital will get higher appraisal from patients and communities, which will give them further enhance their reputation.

Among the hospitals surveyed, about $60 \%$ engaged nursing students in clinical experiences at the hospital but only $40 \%$ of the hospitals employed new graduates registered nurse. Clearly, more needs to be done to engage nursing students, thus increasing employment statistics. The student's clinical experience should be enjoyable and students should feel cared for and valued. Moreover, it is necessary to make effective plans in education so that the practicing student will understand how attractive psychiatric nursing can be.

In order to ensure the quality of psychiatric nursing in Japan, it is necessary to develop an education system in cooperation with hospitals 
Citation: Kataoka M, Miyagawa M, Fuji S, Ito H, Tanioka T, et al. (2016) Characteristics of Psychiatric Hospital Work Environment Found Attractive by Professional Nurse Administrators in Japan. Int J Nurs Clin Pract 3: 207. doi: https://doi.org/10.15344/2394-4978/2016/207

Page 9 of 9

in the community, to enhance the work culture and to implement factors which have been found to be appealing characteristic of psychiatric hospitals.

There were 18 items excluded in the factor analysis: traffic accessibility, web page, electronic health record system, career fair, child-support system, turnover rate, male staff, personal relationship in an office, maintenance of hospital lodgments, maintenance of childcare centers (nursery), exterior view/interior view of hospitals, name recognition of hospital, visits to nursing schools, information exchanged with teachers, working overtime, publicity activity for recruitment of nursing staff at lectures in nursing schools, and publicity activity for recruitment of nursing staffs at the practical training. However, these excluded items also contained important ones which were thought to be tasks for the factor analysis.

One limitation of the study was the sample which only included nurse administrators. Further studies are needed to explore the responses of the staff nurses.

\section{Conclusions}

Nurse administrators of psychiatric hospitals who employ new graduate nurses thought that their hospitals' attractive points were: educational system, support system for acquisition of certificate of clinical nurse specialist/certified nurse, reputation at hospitals as places of employment, knowledge of nursing philosophy and of culture of working places, teaching for students' clinical practice, and salary and other allowances. In addition, the nurse administrators who had no difficulty recruiting nursing staff, thought that their hospital environment had good working hours and leave privileges.

The challenge of nurse staffing of psychiatric hospitals may continue, but this study has furthered the understanding of factors which are considered attractive characteristics of psychiatric hospitals.

\section{Competing Interests}

The authors declare that they have no competing interests.

\section{Acknowledgment}

We would like to express our deep gratitude to the Japanese nursing administrators who participated in this study. And we also would like to express our deep gratitude to the academic supervisors.

\section{References}

1. Oulton JA (2006) The global nursing shortage: an overview of issues and actions. Policy Polit Nurs Pract 7: 34S-39S.

2. May JH, Bazzoli GJ, Gerland AM (2006) Hospitals' responses to nurse staffing shortages. Health Aff (Millwood) 25: W316-323.

3. Carayon P, Gurses AP (2008) Nursing Workload and Patient Safety-A Human Factors Engineering Perspective. In: Hughes RG, editor. Patien Safety and Quality: An Evidence-Based Handbook for Nurses. Rockville, MD: Agency for Healthcare Research and Quality, USA: Chapter 30.

4. Buerhaus PI, Donelan K, Ulrich BT, Norman L, DesRoches C, et al (2007) Impact of the nurse shortage on hospital patient care: comparative perspectives. Health Aff (Millwood) 26: 853-862.

5. Muramatsu N, Akiyama $\mathrm{H}$ (2011) Japan: super-aging society preparing for the future. Gerontologist 51: 425-432.

6. National Institute of Population and Social Security Research (2012) Population Projections for Japan: 2011-2060 (With Long-Range Population Projections: 2061-2110). Tokyo: National Institute of Population and Social Security Research.
7. Ministry of health, labour and welfare (2015) Annual Health, Labour and Welfare Report, Consideration of a depopulating society.

8. http://www.ipss.go.jp/s-info/e/ssj2014/PDF/ssj2014.pdf

9. Japanese Nursing Association (2010) Working conditions in Japan.

10. Han H (2012) The Study on the Actual Conditions of the Nurse Shortage in Japan. Journal of East Studies 10: 1-24

11. Nurses and comedical Committee (2014) Nursing staff retirement and recruitment survey results in 2008. Journal of Japanese Association of Psychiatric Hospitals 28: 66-72.

12. Kataoka M, Miyagawa M, Kuwamura Y, Yasuhara Y, Tanioka T,et al. (2015) Employment of Newly Graduated Nurses by Psychiatric Hospitals in Shikoku region. Shikoku Acta Medica, 71: 23-28 (in Japanese).

13. Hagerty BM, Bissonnette T, Bostrom A, Lovell B, Sieloff C (1995) The status of psychiatric nursing: characteristics and perceptions of nurses in the state of Michigan. Issues Ment Health Nurs 16: 419-432.

14. Meehan T, Boateng A (1997) Crisis intervention workers in New South Wales: knowledge, skills, qualities and preparation. Aust N Z J Ment Health Nurs 6: $122-128$

15. Givens SE, Skully C, Bromley G (2016) Psychiatric Inpatient Bedside Handoff: Implementation of a Quality Improvement Project and Nurses' Responses. J Psychosoc Nurs Ment Health Serv 54: 33-37.

16. Barloon LF, Hilliard W (2016) Legal Considerations of Psychiatric Nursing Practice. Nurs Clin North Am 51: 161-171.

17. Ben Natan M, Drori T, Hochman O (2015) Associative stigma related to psychiatric nursing within the nursing profession. Arch Psychiatr Nurs 29: 388-392.

18. Patterson C, Curtis J, Reid A (2008) Skills, knowledge, and attitudes expected of a newly-graduated mental health nurse in an inpatient setting Int J Ment Health Nurs 17: 410-418.

19. Alhadidi MM, Abdalrahim MS, Al-Hussami M (2016) Nurses' caring and empathy in Jordanian psychiatric hospitals: A national survey. Int J Ment Health Nurs 25: 337-345.

20. Loukidou E, loannidi V, Kalokerinou-Anagnostopoulou A (2010) Institutionalized nursing staff: planning and developing a specialized educational framework that enhances psychiatric nurses' roles and promotes de-institutionalization. J Psychiatr Ment Health Nurs 17: 829-837.

21. Shelton D (2003) The clinical practice of juvenile forensic psychiatric nurses. J Psychosoc Nurs Ment Health Serv 41: 42-53.

22. Handwerker SM. (2012) Transforming nursing education: a review of current curricular practices in relation to Benner's latest work. International Journal of Nursing Education Scholarship 12: 9.

23. Gobet F, Chassy P (2008) Towards an alternative to Benner's theory of expert intuition in nursing: a discussion paper. Int J Nurs Stud 45: 129-139.

24. Benner $P$ (2004) Designing formal classification systems to better articulate knowledge, skills, and meanings in nursing practice. Am J Crit Care 13 426-430.

25. Nigoshima S, Kawakami J, Satake M, Niiduma M (2014) Job Hunting Survey on Newly Employed Nurses in a General Acute Care Hospital. Medical journal of Mutual Aid Association. 63: 60-65.

26. Miyazaki S (2012) Empirical analysis of work consciousness in Japanese female nurses. Journal of the Japan Society for Healthcare administration 49: 19-29.

27. Ministry of health, labour and welfare.

28. Langan JC, Tadych RA, Kao CC (2007) Exploring incentives for RNs to return to practice: a partial solution to the nursing shortage. J Prof Nurs 23: $13-20$ 